\title{
Effects of supplementation with branched chain amino acids and ornithine aspartate on plasma ammonia and central fatigue during exercise in healthy men
}

\author{
Tomasz Mikulski ${ }^{1}$, Jan Dabrowski ${ }^{1}$, Wojciech Hilgier ${ }^{2}$, Andrzej Ziemba ${ }^{1}$, Krzysztof Krzeminski ${ }^{1}$ \\ ${ }^{1}$ Department of Applied Physiology, Mossakowski Medical Research Centre, Polish Academy of Sciences, Warsaw, \\ ${ }^{2}$ Department of Neurotoxicology, Mossakowski Medical Research Centre, Polish Academy of Sciences, Warsaw, Poland
}

\begin{abstract}
Introduction: Our previous studies showed only slight improvement in central fatigue, measured indirectly by psychomotor performance, after branched chain amino acids (BCAA) supplementation during various efforts in healthy men. It is hypothesised that hyperammonaemia resulting from amino acids metabolism may attenuate their beneficial effect on psychomotor performance; therefore, the L-ornithine L-aspartate $(O A)$ as an ammonia decreasing agent was used. The aim of this study was to investigate the effectiveness of oral BCAA+OA supplementation to reduce plasma ammonia concentration and enhance psychomotor performance during exhaustive exercise in healthy men. Material and methods: Eleven endurance-trained men (mean age $32.6 \pm 1.9$ years) performed two sessions (separated by one week) of submaximal cycloergometer exercise for 90 minutes at $60 \%$ of maximal oxygen uptake followed by graded exercise until exhaustion with randomised, double-blind supplementation with a total of $16 \mathrm{~g} B C A A$ and $12 \mathrm{~g} O A$ (BCAA + OA trial) or flavoured water (placebo trial). Before exercise, during both efforts and after 20 minutes of recovery multiple choice reaction time (MCRT), perceived exertion, heart rate and oxygen uptake were measured and venous blood samples were taken for plasma leucine, valine, isoleucine, ornithine, aspartate, free tryptophan (FTRP), ammonia, lactate and glucose determination.

Results: After ingestion, during both efforts and after 20 minutes of recovery the plasma concentrations of all supplemented amino acids were significantly increased, while the fTRP/BCAA ratio decreased in the BCAA + OA trial more than in the placebo trial. At the end of graded exercise plasma fTRP was lower and MCRT shorter in BCAA+OA than in the placebo trial $(p<0.05)$. At the end of prolonged exercise the plasma ammonia concentration was higher in $B C A A+O A$ than in placebo trial $(p<0.05)$. Decreases in plasma ammonia during recovery were significantly higher in $B C A A+O A$ than in the placebo trial. Plasma ammonia positively correlated with the total plasma BCAA and MCRT only in the BCAA + OA trial. The fTRP/BCAA ratio positively correlated with MCRT only in the placebo trial.

Conclusions: Supplementation with $B C A A$ and $O A$ is a useful way to improve MCRT during high-intensity exercise and accelerate the elimination of ammonia at the recovery stage after exercise in healthy young men.
\end{abstract}

Key words: hyperammonaemia, tryptophan, brain, psychomotor performance, reaction time, effort. 


\section{Introduction}

Exercise fatigue usually involves both peripheral muscle and central fatigue. Peripheral fatigue is relatively well understood, while the knowledge of mechanisms underlying central fatigue is still limited. Stress central fatigue may arise from changes in various neurotransmitters in the brain secondary to the changes that occur in the body, especially in the working muscles and in the mind. There are several theories concerning central fatigue, including modulation of glutamatergic and GABA-ergic synaptic signal transmission, as well as increased serotonin, which works as a neurotransmitter in the brain $[3,26]$. Since administration of selective serotonin reuptake inhibitors in humans has shown increased fatigue and perceived exertion $[41,49]$ it seems likely that central fatigue may result from enhanced synthesis and release of serotonin from some neurons in the brain $[14,26,50]$. Serotonin is synthesised from L-tryptophan by a metabolic pathway consisting inter alia of tryptophan hydroxylase, the rate-limiting enzyme of serotonin synthesis [27,32,37]. Tryptophan circulates in blood in both albumin-bound and free forms. The free form crosses the blood-brain barrier and is converted into serotonin. Increased plasma free tryptophan (fTRP) enhances the rate of entry of tryptophan into the brain. Free tryptophan, the branchedchain amino acids (BCAA), as well as other amino acids share a common transporter across the bloodbrain barrier $[17,28,38]$. Similarly, tryptophan and free fatty acids (FFA) compete for the same binding sites to albumin [24].

Thus, the reduction in BCAA and elevation in FFA may lead to an increase of tryptophan uptake into the brain. Some studies in human subjects [19] and in animals $[13,37]$ have shown that exercise-induced increases in fTRP/BCAA ratio, due to higher BCAA oxidation in skeletal muscles and FFA mobilisation from adipose tissue, are associated with an increased rate of serotonin synthesis in brain neurons $[12,49]$. The rate of serotonin synthesis can be lowered by raising the blood BCAA concentration, which reduces tryptophan uptake into the brain. Studies in human subjects have shown that ingestion of BCAA reduces the perceived exertion and mental fatigue during standardised cycle ergometer exercise and sustained heavy exercise (a competitive 30-km cross-country race), as well as improves cognitive performance after exercise in human subjects [12,15,16,29,39,49]. However, the BCAA supplementation did not affect physical fatigue and was ineffective in preventing muscle power loss and perceived muscle pain during strenuous exercise such as in a marathon race $[5,49]$. Our previous studies in soccer players $[39,54]$ showed a slight improvement in psychomotor performance after BCAA supplementation during both graded exercise until exhaustion and treadmill running simulating a football game.

Several studies demonstrated that both ingested BCAA and prolonged exercise itself can enhance the circulating level and the cerebral uptake of ammonia $[4,25,40]$. Strüder et al. [49] demonstrated that the increase in plasma ammonia during strenuous activity is significantly higher in subjects supplemented with BCAA than in the group supplemented with tyrosine or placebo. Elevated ammonia concentration is a result of both increased catabolism of amino acids and purine nucleotide deamination within the working muscles $[34,43]$. Since ammonia is crossing the blood-brain barrier [6], its high circulating level can cause detrimental effects on neurotransmitter metabolism (glutamate, GABA), neurotransmission as well as cerebral metabolism, which in turn may lead to central fatigue $[3,4,9]$. The enhanced cerebral ammonia uptake was observed during both prolonged and maximal exercise [4,9]. Thus, it was concluded that hyperammonaemia may cause central fatigue. Since in most experimental studies $[7,35,42,44,48]$ the greatest effect on ammonia reduction was achieved with L-ornithine L-aspartate (OA), it seems likely that oral intake of BCAA together with OA may reduce ammonia accumulation and cerebral absorption, thereby delaying fatigue.

The present study was designed to investigate the effectiveness of a BCAA + OA containing drink to reduce plasma ammonia concentration and enhance the psychomotor performance (assessed by measurement of multiple-choice reaction time) during high-intensity exercise in healthy young men. It has been reported that the multiple-choice reaction time closely reflects the flow rate of neurophysiological, cognitive, and information processes that are created by the action of stimulus on the subject's sensory system $[10,30]$.

\section{Material and methods}

Eleven healthy, endurance-trained male volunteers (mean age $32.6 \pm 1.9$ years, height $180 \pm 2.0 \mathrm{~cm}$, weight $73.6 \pm 1.6 \mathrm{~kg}$, BMI $22.3 \pm 0.3 \mathrm{~kg} \times \mathrm{m}^{-2}$ (range 20.60-24.19 kg $\times \mathrm{m}^{-2}$ ), peak oxygen uptake $59 \pm 1 \mathrm{ml} \times$ 
$\mathrm{kg}^{-1} \times \mathrm{min}^{-1}$ (range 56-73 $\mathrm{ml} \times \mathrm{kg}^{-1} \times \mathrm{min}^{-1}$ ) and mean arterial blood pressure $79 \pm 3 \mathrm{mmHg}$; the values are means \pm SEM) participated in this study. They were asked to refrain from strenuous activity and caffeine or alcohol consumption three days prior to the study. All subjects gave their informed consent to participate in the study. The investigation conforms to the principles outlined in the Declaration of Helsinki and was approved by the Local Ethics Committee (permission No. KB/48/2009). All procedures were carried out under similar environmental conditions $\left(23-24^{\circ} \mathrm{C}\right.$ and 50-60\% humidity) between 8:00 and 12:00 AM. The subjects visited the laboratory three times with oneweek intervals between the tests.

On the first occasion, the subjects were submitted to an incremental, graded exercise test performed on a bicycle ergometer (EM 840, Siemens, Germany) until volitional exhaustion, to determine their maximal oxygen uptake $\left(\mathrm{VO}_{2}\right.$ max). The workloads were increased by 50 Watts (W) every 3 minutes starting with $50 \mathrm{~W}$. Oxygen uptake, carbon dioxide production and heart rate (HR) were continuously recorded using a $\mathrm{V}_{\max } 29$ (Sensormedics, USA) analyser. Before exercise and during the last two minutes of each workload a multiple-choice reaction time (MCRT) test was conducted in order to familiarise the subjects with the procedure chosen for evaluation of psychomotor performance [10,30,39]. The subjects were asked to react to visual and audio stimuli by pressing as fast as possible the corresponding buttons on the handlebars of the bicycle ergometer. Three coloured lights and sound were emitted in various time intervals by a psychomotor performance measurement device (CRR-APR-2/05, Unipar, Poland) with the signaller placed two metres in front of the subject at eye level. There were 15 stimuli for which the reaction time was recorded in milliseconds (red light for right hand and sound for left hand), while other 15 stimuli (green and orange light) were supposed to be ignored.

On the second and third occasion, according to the same protocol, the subjects were submitted to submaximal exercise performed on a bicycle ergometer lasting 90 minutes at $60 \% \mathrm{HR}_{\max }$ followed by graded exercise performed until volitional exhaustion as during the first visit. Before exercise each subject performed an MCRT test. Afterwards, a catheter was inserted into the antecubital vein in one arm for sampling of the plasma concentrations of amino acids (valine, leucine, isoleucine, tryptophan, ornithine, aspartic acid), ammonia, lactate, and glucose. The blood samples were collected into the tubes containing EDTA as an anticoagulant. Then, the subjects were randomised in a double-blind crossover manner to receive amino acids with ornithine aspartate (BCAA + OA trial, $n=11$ ) or flavoured water as placebo (placebo trial, $n=11$ ). The supplements were given in a cross-over fashion, i.e. six of the subjects started with BCAA and OA and the other five started with placebo, in the second experiment, after one week, the order was changed. Before exercise the subjects ingested $500 \mathrm{ml}$ of either a flavoured solution containing $10 \mathrm{~g}$ of BCAA (leucine, valine, isoleucine; $2: 1: 1$, Olimp Labs) with $6 \mathrm{~g}$ of OA (Hepa-Merz 3000, Merz Pharmaceuticals GmbH, Germany) or flavoured water. Both drinks contained artificial sweetener, fruit flavour - orange syrup mixed with citric acid - and were indistinguishable in taste.

After a 30-minute rest period in a sitting position, baseline blood sample was taken and the subjects were submitted to prolonged submaximal exercise for 90 minutes at $60 \%$ of $\mathrm{HR}_{\max }$.

In the $30^{\text {th }}$ minute of exercise the second portion of $500 \mathrm{ml}$ solution containing $6 \mathrm{~g}$ of BCAA and $6 \mathrm{~g}$ of OA or placebo was administered. After three minutes of rest, upon completion of the prolonged exercise, the subjects started graded exercise until volitional exhaustion, as they did at the first visit. Blood samples for determinations of the plasma amino acids, ammonia, glucose and lactate concentrations were taken in the $45^{\text {th }}$ minute and at the end of prolonged exercise as well as at the end of each workload of graded exercise until exhaustion and after 20 minutes of recovery. Before exercise, in the $45^{\text {th }}$ minute and at the end of prolonged exercise, as well as during last two minutes of each workload of graded exercise until exhaustion MCRT was measured. Additionally, at the end of each workload of graded exercise the rate of perceived exertion with a 20-point Borg scale was recorded.

Amino acids were assayed in deproteinised blood samples using HPLC with fluorescence detection after derivatisation in a timed reaction with o-phthalaldehyde plus mercaptoethanol, as described by Kilpatrick [36]. Derivatised samples were injected onto a $150 \times 4.6-\mathrm{mm} 5-\mu$ Hypersil Gold BDS C18 column with a mobile phase of 50-mM phosphate buffer containing 10\% v/v methanol, pH 6.2 (solvent A), and methanol (solvent B). Blood ammonia and lactate concentrations were analysed using a photometer Dr Lange LP420 (Konisburg, Germany) and cuvette 
tests AM1054 (Randox, UK) and LKM140 (Hach Lange, Germany), respectively. Blood glucose concentration was measured using a glucometer (Optium Xido, UK). The fTRP/BCAA ratio was calculated by dividing the concentration of free tryptophan by the summarised concentrations of valine, leucine, and isoleucine (expressed in the same units).

\section{Statistical analysis}

The data are expressed as means with standard errors of the mean (SEM). Distribution of results was analysed by Shapiro-Wilk's test and paired $t$ test or Wilcoxon test was used to compare results, accordingly. Changes over time were examined with twoway ANOVA for repeated measures, and $p<0.05$ was accepted as the level of significance. In addition, correlation coefficients were calculated between variables using linear regression analysis. For calculations, Statistica (2001) version 6 (Statsoft Inc., Tulsa, OK, USA) was used.

\section{Results}

The aerobic capacities of the young men were within the upper normal range for men of their res- pective ages. There were no significant differences in $H R$, oxygen uptake and rating of perceived exertion, as well as in blood lactate or glucose between BCAA $+O A$ and placebo trials at all stages of the study. Supplementation with BCAA + OA caused significant increases in the pre-exercise resting plasma concentrations of valine, leucine, isoleucine and ornithine. The concentration of these amino acids in the plasma remained significantly elevated during both types of exercise (prolonged - of moderate intensity and graded - until exhaustion) as well as after 20 minutes of the recovery period (Table I and II). Plasma aspartic acid concentration was significantly higher at the end of prolonged moderate-intensity exercise and after 20 minutes of recovery period only in the BCAA + OA trial (Table II). At the end of graded exercise until exhaustion plasma tryptophan was significantly lower in the BCAA + OA than in the placebo trial $(19.2 \pm 2.7 \mu \mathrm{M}$ vs. $29.1 \pm 2.0 \mu \mathrm{M}, p<0.05)$. The ratio of fTRP/BCAA was significantly lower in the BCAA + OA than in the placebo trial at all stages of the study (Table II). At the end of prolonged submaximal exercise the plasma ammonia concentration was significantly higher in the BCAA + OA than in the placebo trial (Fig. 1). Plasma ammonia decrease after graded exer-

Table I. Plasma valine, leucine, and isoleucine concentrations (branched chain amino acids - BCAA) at rest and during prolonged exercise followed by graded exercise until exhaustion with or without BCAA and ornithine aspartate (OA) supplementation

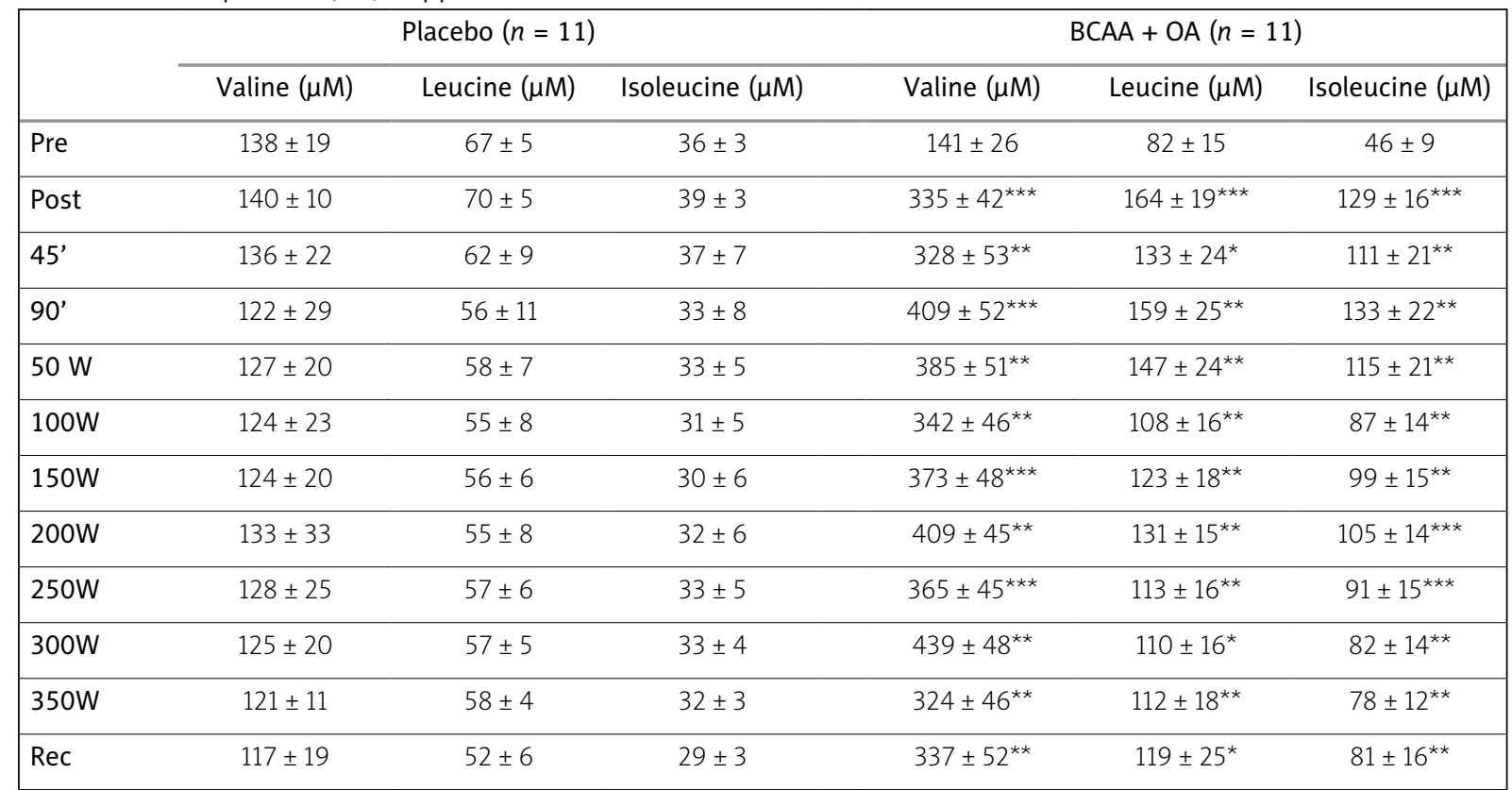

Pre - rest, Post - 30 minutes after ingestion of BCAA and OA (BCAA+OA) or placebo, 45' and 90'- minutes during prolonged submaximal exercise, 50-350 Watts - workloads of graded exercise until exhaustion, Rec - at the $20^{\text {th }}$ min of the recovery period, $B C A A+O A-$ amino acids with ornithine aspartate trial. Values are means \pm SEM. ${ }^{*}$ - denote significant differences between trials: ${ }^{*} p<0.05,{ }^{* *} p<0.01,{ }^{* * *} p<0.001$ 
Table II. Plasma ornithine and aspartic acid concentrations and the ratio of free tryptophan to branched chain amino acids (fTRP/BCAA) at rest and during prolonged exercise followed by graded exercise until exhaustion with or without BCAA and ornithine aspartate (OA) supplementation

\begin{tabular}{|c|c|c|c|c|c|c|}
\hline & \multicolumn{3}{|c|}{ Placebo $(n=11)$} & \multicolumn{3}{|c|}{$\mathrm{BCAA}+\mathrm{OA}(n=11)$} \\
\hline & Ornithine $(\mu M)$ & Aspartic acid $(\mu M)$ & fTRP/BCAA & Ornithine $(\mu \mathrm{M})$ & Aspartic acid $(\mu M)$ & fTRP/BCAA \\
\hline Pre & $48 \pm 6$ & $3.3 \pm 0.7$ & $0.137 \pm 0.012$ & $47 \pm 7$ & $4.0 \pm 0.8$ & $0.134 \pm 0.013$ \\
\hline Post & $49 \pm 6$ & $2.8 \pm 0.5$ & $0.137 \pm 0.013$ & $107 \pm 14^{\star \star *}$ & $3.4 \pm 0.6$ & $0.056 \pm 0.006^{* * *}$ \\
\hline $45^{\prime}$ & $45 \pm 6$ & $2.5 \pm 0.5$ & $0.149 \pm 0.015$ & $119 \pm 22^{* *}$ & $3.1 \pm 0.5$ & $0.053 \pm 0.010^{\star \star * *}$ \\
\hline $90^{\prime}$ & $41 \pm 5$ & $2.5 \pm 0.4$ & $0.155 \pm 0.017$ & $187 \pm 31^{\star * *}$ & $4.5 \pm 0.8^{*}$ & $0.047 \pm 0.010^{\star * \star}$ \\
\hline $50 \mathrm{~W}$ & $41 \pm 5$ & $3.1 \pm 0.5$ & $0.139 \pm 0.014$ & $155 \pm 27^{\star * *}$ & $4.4 \pm 0.9$ & $0.047 \pm 0.011^{* *}$ \\
\hline $100 \mathrm{~W}$ & $40 \pm 5$ & $2.9 \pm 0.4$ & $0.151 \pm 0.016$ & $124 \pm 18^{* * *}$ & $3.5 \pm 0.7$ & $0.056 \pm 0.140^{* *}$ \\
\hline $150 \mathrm{~W}$ & $40 \pm 5$ & $2.7 \pm 0.5$ & $0.148 \pm 0.009$ & $134 \pm 18^{* * *}$ & $3.7 \pm 0.7$ & $0.055 \pm 0.019^{\star *}$ \\
\hline $200 \mathrm{~W}$ & $39 \pm 6$ & $2.9 \pm 0.5$ & $0.151 \pm 0.015$ & $147 \pm 20^{\star * *}$ & $4.0 \pm 0.8$ & $0.051 \pm 0.012^{\star \star}$ \\
\hline $250 \mathrm{~W}$ & $41 \pm 6$ & $2.6 \pm 0.4$ & $0.148 \pm 0.016$ & $122 \pm 18^{* * *}$ & $3.7 \pm 0.7$ & $0.058 \pm 0.017^{\star *}$ \\
\hline $300 \mathrm{~W}$ & $44 \pm 6$ & $3.0 \pm 0.7$ & $0.139 \pm 0.011$ & $112 \pm 21^{\star *}$ & $3.5 \pm 0.7$ & $0.052 \pm 0.011^{\star \star}$ \\
\hline $350 \mathrm{~W}$ & $42 \pm 5$ & $2.9 \pm 0.6$ & $0.144 \pm 0.014$ & $102 \pm 15^{* *}$ & $4.0 \pm 0.6$ & $0.049 \pm 0.014^{\star *}$ \\
\hline $\operatorname{Rec}$ & $42 \pm 7$ & $2.1 \pm 0.5$ & $0.136 \pm 0.012$ & $98 \pm 21^{*}$ & $3.7 \pm 0.6^{\star \star \star}$ & $0.058 \pm 0.015^{\star *}$ \\
\hline
\end{tabular}

Pre - rest, Post - 30 minutes after ingestion of BCAA and $O A$ (BCAA+OA) or placebo, 45' and 90'- minutes during prolonged submaximal exercise, $50-350$ Watts - workloads of graded exercise until exhaustion, Rec - at the $20^{\text {th }}$ min of the recovery period, $B C A A+O A$ - amino acids with ornithine aspartate trial. Values are means \pm SEM. ${ }^{*}$ - denote significant differences between trials: ${ }^{*} p<0.05,{ }^{* *} p<0.01,{ }^{* * *} p<0.001$

cise until exhaustion was significantly higher in the $\mathrm{BCAA}+\mathrm{OA}$ than in the placebo trial $(\Delta 99.5 \pm 16.6 \mu \mathrm{M}$ vs. $\Delta 51.4 \pm 11.5 \mu \mathrm{M}, p<0.05$; Fig. 2). After 20 minutes of the recovery period the plasma ammonia concentration did not differ significantly from the resting values only in the BCAA + OA trial (Fig. 1). At work-

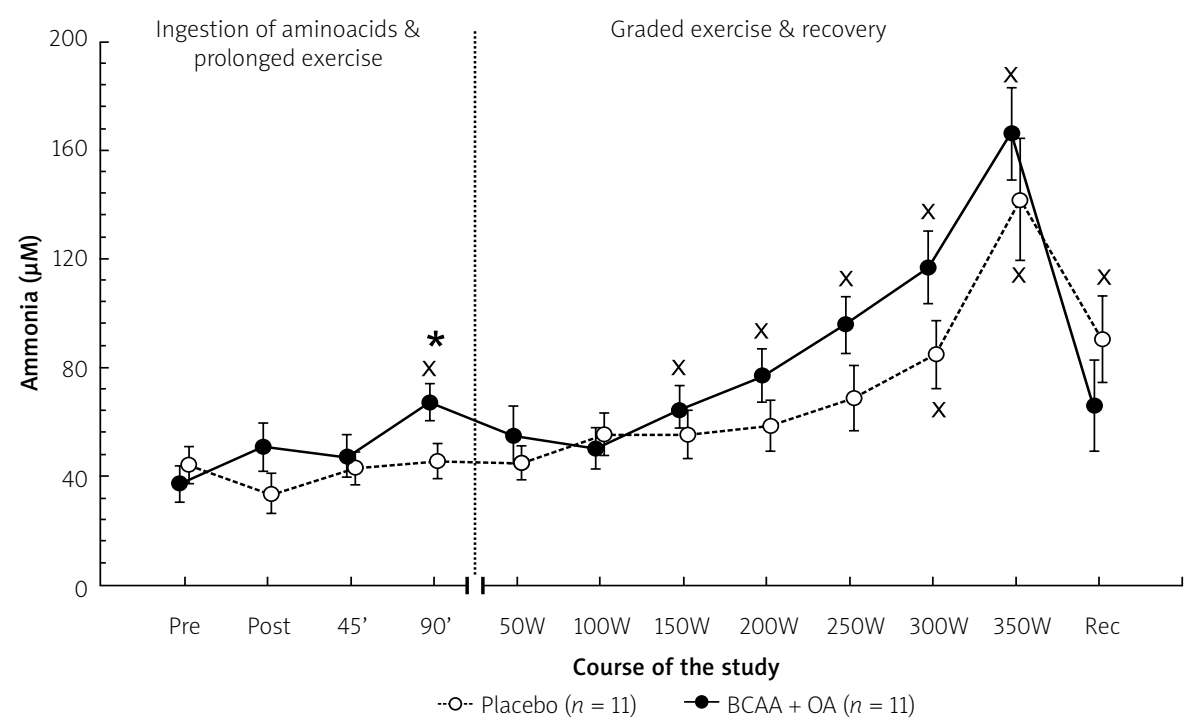

Fig. 1. Plasma ammonia concentration at rest (Pre) 30 minutes after ingestion of branched chain amino acids $(B C A A)$ and ornithine aspartate (OA) (BCAA + OA) or placebo (Post), during both prolonged submaximal exercise (45' and 90') and graded exercise until exhaustion (50-350 Watts workloads) and at the $20^{\text {th }}$ min of the recovery period (Rec). Values are means $\pm \mathrm{SE}$. * - denotes significant differences between trials: ${ }^{*} p<0.05$; $x$ - denotes significant differences from the resting values: ${ }^{x_{p}}<0.05$. 


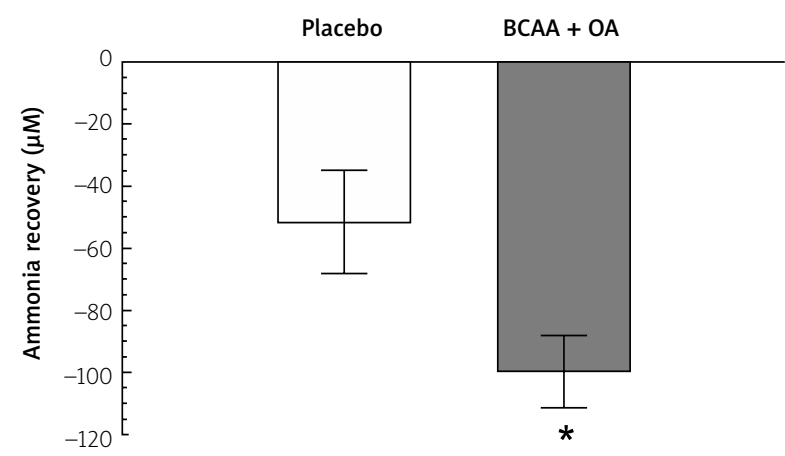

Fig. 2. Restitution of plasma ammonia concentration after graded exercise in placebo and amino acids-supplemented (branched chain amino acids and ornithine aspartate; $B C A A+O A$ ) trials. $\Delta$ - the difference between concentration achieved at the end of maximal exercise and after 20 minutes of recovery. Values are means $\pm S E$. * - denote significant differences between trials: ${ }^{*} p<0.05$.

loads of $100 \mathrm{~W}, 150 \mathrm{~W}$ and at the end of graded exercise until exhaustion MCRT was significantly shorter in the BCAA + OA than in the placebo trial $(272 \pm 8 \mathrm{~ms}$ vs. $286 \pm 8 \mathrm{~ms}, p<0.05 ; 271 \pm 9 \mathrm{~ms}$ vs. $287 \pm 8 \mathrm{~ms}, p<0.05$; and $281 \pm 7 \mathrm{~ms}$ vs. $310 \pm 8 \mathrm{~ms}$, $p<0.01$, respectively; Fig. 3). Plasma ammonia concentration correlated positively with the total plasma BCAA in both trials, and with MCRT only in the
BCAA + OA trial $(r=0.40, p<0.05 ; r=0.20, p<0.05$; $r=0.35 ; p<0.05$, respectively). The fTRP/BCAA ratio correlated positively with MCRT only in the placebo trial ( $r=0.38, p<0.05$; Fig. 4).

\section{Discussion}

The new finding of this study is that, despite similar levels of ammonia in both trials, shorter multiple-choice reaction time at the end of strenuous, exhaustive exercise and faster return of ammonia concentration to the pre-exercise resting values were observed in the BCAA + OA trial. Furthermore, significant positive correlations were found between plasma ammonia and the total plasma BCAA concentration in both trials and between plasma ammonia and MCRT in the BCAA + OA trial. Similar correlation was found between the fTRP/BCAA ratio and MCRT only in the placebo trial. The study showed also that $B C A A+O A$ supplementation, although effective in increasing blood concentrations of BCAA and OA, did not influence aerobic performance or the rating of perceived exertion.

Several studies showed that ammonia is tightly regulated in response to exercise, being affected by factors such as exercise duration and intensity and energy substrates availability $[4,9,40]$. During highintensity or exhaustive exercise ammonia is mainly

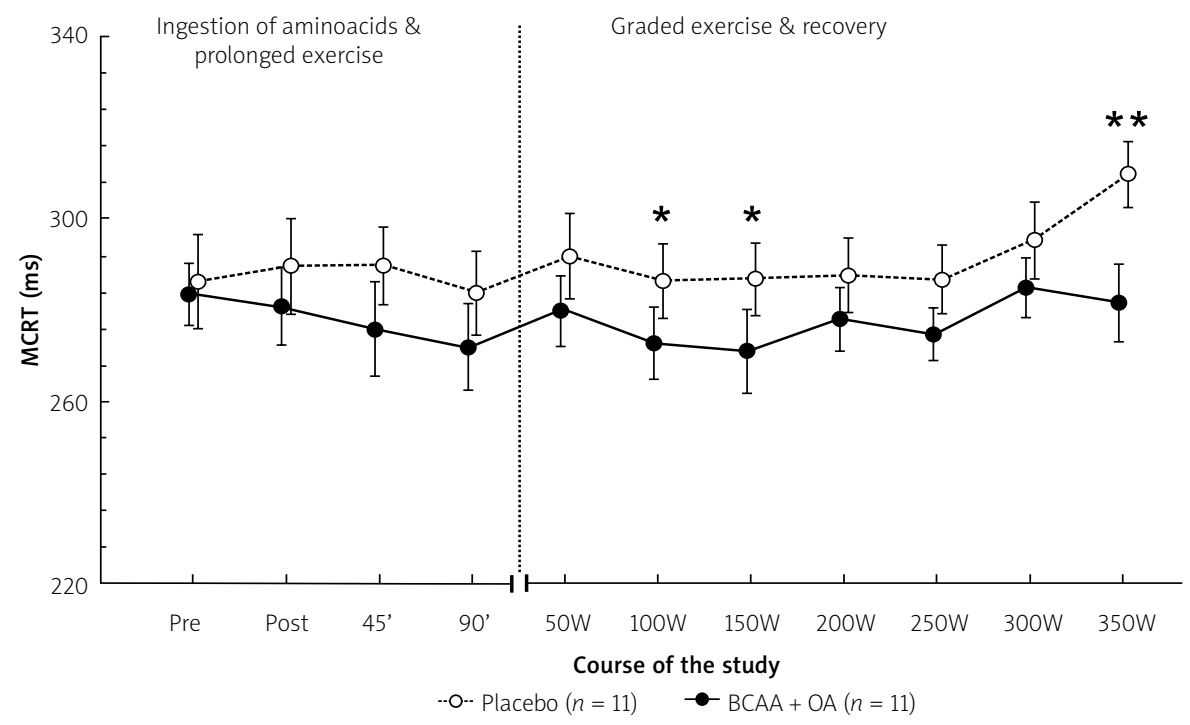

Fig. 3. Multiple choice reaction time (MCRT) at rest (Pre) 30 minutes after ingestion of branched chain amino acids (BCAA) and ornithine aspartate (BCAA + OA) or placebo (Post), during both prolonged submaximal exercise (45' and 90') and graded exercise until exhaustion (50-350 Watts workloads) and at the $20^{\text {th }}$ min of the recovery period $(\operatorname{Rec})$. Values are means $\pm \mathrm{SE} .{ }^{*}-$ denotes significant differences between trials: ${ }^{*} p<0.05,{ }^{* *} p<0.01$. 


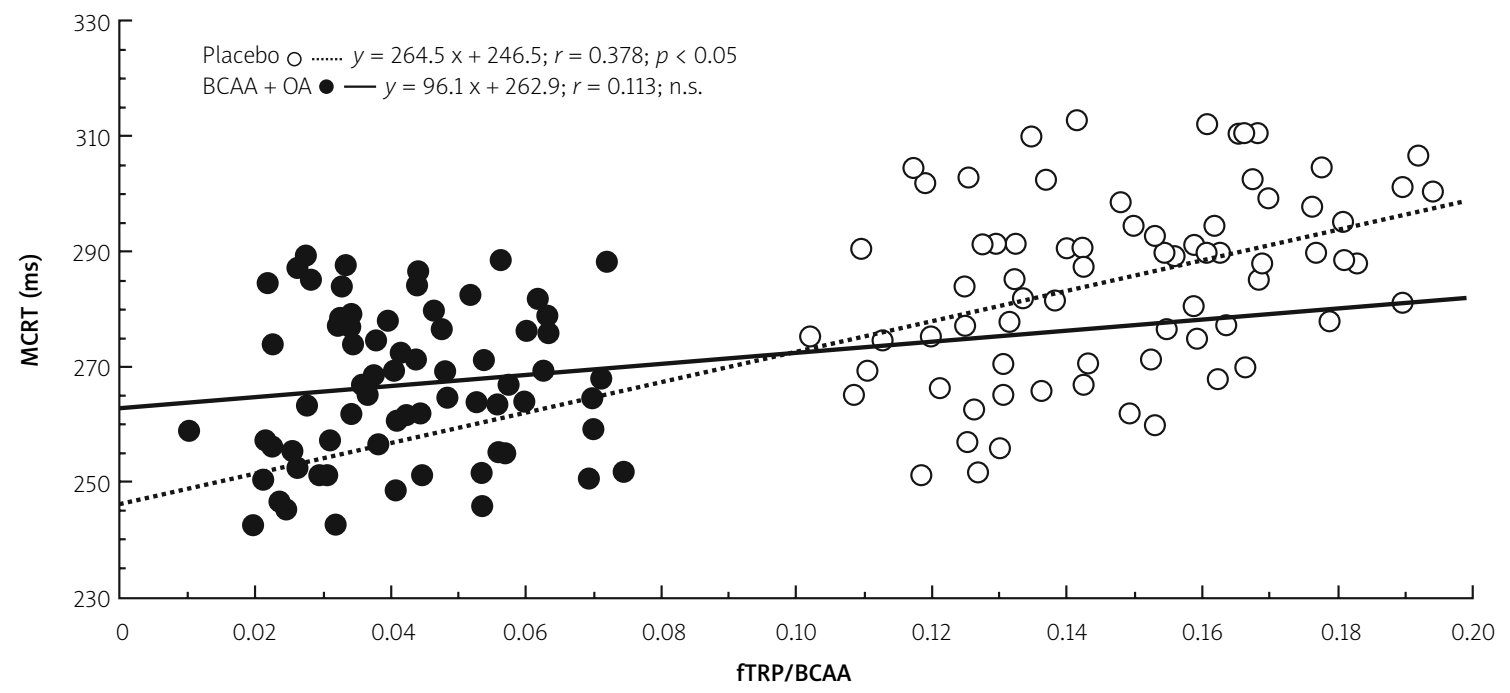

Fig. 4. Correlation between plasma free tryptophan to branched chain amino acids ratio (fTRP/BCAA) and multiple-choice reaction time (MCRT) during exercise in placebo and amino acids supplemented (branched chain amino acids and ornithine aspartate; BCAA + OA) trials.

produced by deamination of AMP in the purine nucleotide cycle, which takes place in the contracting muscles.

The relationship between plasma ammonia and total plasma BCAA observed in this study may be at least partly associated with deamination of the branched chain amino acids for additional energy provision. Based on previous studies [51,52], it might be speculated that increased oxidation of BCAA limited the tricarboxylic acid cycle activity and fatty acid oxidation, and therefore plays an important role in fatigue mechanisms during prolonged exercise, leading to glycogen depletion at intensities above $60 \%$ $\mathrm{VO}_{2}$ max. Nybo et al. [40] demonstrated that high levels of systemic ammonia during prolonged exercise enhanced the uptake of ammonia by the brain in endurance-trained male subjects. Positive correlations were observed between the arterial ammonia concentration and both the cerebral uptake and the cerebrospinal fluid ammonia. Weaker correlation was found between the cerebrospinal fluid ammonia and perceived exertion at the end of prolonged exercise (at $60 \%$ of $\mathrm{VO}_{2} \max$ ). The authors suggested that exercise-induced hyperammonaemia may induce a transient state of acute ammonia toxicity with concomitant disturbances in brain neurotransmitters, leading to central fatigue. The findings obtained in trained rats showed that an enhanced brain ammonia level evoked by exhaustive exercise stimulates glutamine synthesis as a result of detoxification and subsequently decreases both brain glutamate levels in all brain structures and gamma-aminobutyric acid (GABA) levels in the striatum. Thus, changes in neurotransmitters metabolism and subsequent central fatigue depend on the effect of ammonia on the excitatory amino acids [31]. Although the weak correlation between plasma ammonia concentration and MCRT in the BCAA + OA trial observed in the present study supports this mechanism, shortened MCRT at the end of exhaustive exercise in the BCAA + OA trial indicates that other factors are involved in the development of central fatigue.

An additional finding of the present investigation is that in the BCAA + OA trial the ratio of fTRP/ $B C A A$ was significantly lower than in placebo trial at all stages of the study. Since tryptophan was not administered, the changes in $\mathrm{FTRP/BCAA}$ ratio resulted from the BCAA supplementation [28,32], which prevented the described decrease of these amino acids in the latter stages of exercise $[15,18,28]$. However, the present study has not confirmed such a decrease that should have been observed in the placebo trial. The decrease in the fTRP/BCAA ratio would lead to decreased tryptophan transport across the blood-brain barrier because BCAA and tryptophan compete for entry by the same large neutral amino acid transporter [15-17]. This should lead to a lower level of 5-hydroxytryptamine responsible for 
central fatigue $[15,23,50]$. Thus, the ingestion of BCAA could reduce the exercise-induced increase in brain tryptophan uptake and delay fatigue. The shortening of the multiple-choice reaction time at the end of exhaustive exercise in the BCAA + OA trial observed in the present study supports this "central fatigue" hypothesis.

However, it is unclear why the ITRP/BCAA ratio did not correlate with MCRT in the BCAA + OA trial. In this regard, it seems worth mentioning that MCRT reflects not only one pathway of the selected neurotransmitter or the flow rate of neurophysiological and information processes, but also several cognitive modalities (attention, impulse control, processing speed, cognitive flexibility) that are created by the action of stimulus on the subject's sensory system $[10,30]$. During heavier but still submaximal workloads other involved pathways might dominate, for example BCAA also competitively inhibits tyrosine uptake into the brain, and thus catecholamine synthesis, which reduce physical performance [23].

In the placebo trial reflecting the physiological concentrations of amino acids and the balance in nitrogen turnover, MCRT correlated with FTRP/BCAA ratio and probably was not influenced by ammonia (Fig. 4). In the BCAA + OA trial this relationship disappeared, and enhanced nitrogen turnover adversely affected psychomotor performance, because MCRT became slower with increased blood ammonia concentration, which was not diminished during exercise by $\mathrm{OA}$. This finding suggests that exogenous amino acids can easily exceed autoregulatory capacity, disturb nitrogen homeostasis and contribute to the development of central fatigue. Thus, very careful selection of doses of BCAA should be applied in order to shift the fTRP/BCAA ratio but not to increase the nitrogen turnover beyond the limits resulting in significantly enhanced ammonia production.

Administration of OA prevented the expected increase in blood ammonia concentration after the BCAA supplementation during the graded exhaustive exercise and therefore probably prevented the deterioration of multiple choice reaction time usually occurring in the final stage of the exhaustive exercise [20-22,39,54], which reflects improved psychomotor performance (Fig. 3).

During prolonged exercise of constant intensity and graded exercise with subsequent workloads without any breaks the influence of OA on ammonia was limited, possibly due to decreased splanchnic (especially liver) blood flow [46]. The supposed site of detoxification action of OA in hepatocytes [47] by stimulation of urea cycle and glutamine synthesis [33] can be systematically effective only when sufficient blood flow through the liver is maintained. However, in the BCAA + OA trial after 20 minutes of the recovery period a beneficial effect of reduced ammonaemia was observed. It is very likely that the supplemented OA was available for ammonia metabolism in the liver, but the splanchnic blood flow was so limited by exercise that its action could not be significant. After cessation of exercise when liver blood flow increased $[46,53]$ ammonia degradation became significantly faster in BCAA + OA than in the placebo trial. Improved recovery capacity is very important in daily activities or sports with repeated efforts with short breaks between them. On the other hand, application of OA is questionable in high-intensity efforts performed as one bout of exercise without any breaks. In current trends for faster mobilisation of patients suffering from any diseases care should be taken in treatment of patients with hyperammonaemia. Activities of moderate intensity with frequent breaks can be considered, whereas intense exercise, especially of constant intensity and without breaks is not recommended as it can increase ammonia production and impair its breakdown and effectiveness of treatment with ammonia-decreasing drugs acting in the liver, e.g. OA. Supplements based on proteins and amino acids are currently one of the most overused and overdosed, so any recommendations for their use should be calculated very carefully. In certain cases the use of OA seems to be rational and in agreement with the results of most studies $[1,7,8,11,42,44]$; however, some critical works are also present $[2,45]$.

\section{Conclusions}

In summary, this study demonstrated that BCAA + OA supplementation, although effective in increasing blood concentrations of BCAA and OA, did not influence aerobic performance or rating of perceived exertion. The most important finding is that, despite similar levels of ammonia in both trials, shorter multiple-choice reaction time at the end of strenuous exhaustive exercise and faster return to the pre-exercise resting values were observed only in the BCAA + OA trial.

These results suggest that oral supplementation with $B C A A+O A$ delays central fatigue during pro- 
longed exhaustive exercise in endurance-trained young men, but this requires more detailed studies.

\section{Acknowledgments}

The study was financed by the grant of Ministry of Science and Education N N404 051337.

\section{Disclosure}

\section{Authors declare no conflict of interest.}

\section{References}

1. Abid S, Jafri W, Mumtaz K, Islam M, Abbas Z, Shah HA, Hamid S. Efficacy of L-ornithine-L-aspartate as an adjuvant therapy in cirrhotic patients with hepatic encephalopathy. J Coll Physicians Surg Pak 2011; 21: 666-671.

2. Acharya SK, Bhatia V, Sreenivas V, Khanal S, Panda SK. Efficacy of L-ornithine L-aspartate in acute liver failure: a double-blind, randomized, placebo-controlled study. Gastroenterology 2009; 136: 2159-2168.

3. Albrecht J, Norenberg MD. Glutamine: a Trojan horse in ammonia neurotoxicity. Hepatology 2006; 44: 788-794.

4. Ament W, Huizenga JR, Kort E, van der Mark TW, Grevink RG, Verkerke GJ. Respiratory ammonia output and blood ammonia concentration during incremental exercise. Int J Sports Med 1999; 20: 71-77.

5. Areces F, Salinero JJ, Abian-Vicen J, Gonzalez-Millan C, GalloSalazar C, Ruiz-Vicente D, Lara B, Del Coso J. A 7-day oral supplementation with branched-chain amino acids was ineffective to prevent muscle damage during a marathon. Amino Acids 2014; 46: 1169-1176.

6. Bachmann C. Mechanisms of hyperammonemia. Clin Chem Lab Med 2002; 40: 653-662.

7. Bai M, Yang Z, Qi X, Fan D, Han G. L-ornithine-l-aspartate for hepatic encephalopathy in patients with cirrhosis: a meta-analysis of randomized controlled trials. J Gastroenterol Hepatol 2013; 28: 783-792.

8. Bai M, He C, Yin Z, Niu J, Wang Z, Qi X, Liu L, Yang Z, Guo W, Tie J, Bai W, Xia J, Cai H, Wang J, Wu K, Fan D, Han G. Randomised clinical trial: L-ornithine-L-aspartate reduces significantly the increase of venous ammonia concentration after TIPSS. Aliment Pharmacol Ther 2014; 40: 63-71.

9. Banister EW, Cameron BJ. Exercise-induced hyperammonemia: peripheral and central effects. Int J Sports Med 1990; 11: S129-142.

10. Batra A, Vyas S, Gupta J, Gupta K, Hada R. A comparative study between young and elderly indian males on audio-visual reaction time. Indian Journal of Scientific Research and Technology 2014; 2: 25-29.

11. Blanco Vela Cl, Poo Ramírez JL. Efficacy of oral L-ornithine L-aspartate in cirrhotic patients with hyperammonemic hepatic encephalopathy. Ann Hepatol 2011; 10 Suppl 2: S55-59.

12. Blomstrand E, Hassmen P, Ekblom B, Newsholme EA. Influence of ingesting a solution of branched-chain amino acids on perceived exertion during exercise. Acta Physiol Scand 1997; 159: 41-49.
13. Blomstrand E, Perrett D, Parry-Billings M, Newsholme EA. Effect of sustained exercise on plasma amino acid concentrations and on 5-hydroxytryptamine metabolism in six different brain regions of the rat. Acta Physiol Scand 1989; 136: 473-481.

14. Blomstrand E, Saltin B. BCAA intake affects protein metabolism in muscle after but not during exercise in humans. Am J Physiol Endocrinol Metab 2001; 281: E365-374.

15. Blomstrand $E$. A role for branched-chain amino acids in reducing central fatigue. J Nutr 2006; 136: S544-547.

16. Blomstrand E. Amino acids and central fatigue. Amino Acids 2001; 20: 25-34.

17. Boado RJ, Li JY, Nagaya M, Zhang C, Pardridge WM. Selective expression of the large neutral amino acid transporter at the blood-brain barrier. Proc Natl Acad Sci USA 1999; 96: 1207912084.

18. Borgenvik M, Nordin M, Mikael Mattsson C, Enqvist JK, Blomstrand E, Ekblom B. Alterations in amino acid concentrations in the plasma and muscle in human subjects during $24 \mathrm{~h}$ of simulated adventure racing. Eur J Appl Physiol 2012; 112: 3679-3688.

19. Chaouloff F. Effects of acute physical exercise on central serotonergic systems. Med Sci Sports Exerc 1997; 29: 58-62.

20. Chmura J, Krysztofiak H, Ziemba AW, Nazar K, Kaciuba-Uściłko H. Psychomotor performance during prolonged exercise above and below the blood lactate threshold. Eur J Appl Physiol Occup Physiol 1998; 77: 77-80.

21. Chmura J, Nazar K. Kaciuba-Uściłko H. Próg psychomotoryczny zmęczenia. Sport Wyczynowy 2007; 4-6: 508-510.

22. Chmura J, Nazar K. Parallel changes in the onset of blood lactate accumulation (OBLA) and threshold of psychomotor performance deterioration during incremental exercise after training in athletes. Int J Psychophysiol 2010; 75: 287-290.

23. Choi S, DiSilvio B, Fernstrom MH, Fernstrom JD. Oral branchedchain amino acid supplements that reduce brain serotonin during exercise in rats also lower brain catecholamines. Amino Acids 2013; 45: 1133-1142.

24. Curzon G, Friedel J, Knott PJ. The effect of fatty acids on the binding of tryptophan to plasma protein. Nature 1973; 242: 198-200.

25. Dalsgaard MK, Volianitis S, Yoshiga CC, Dawson EA, Secher NH. Cerebral metabolism during upper and lower body exercise. J Appl Physiol 2004; 97: 1733-1739.

26. Davis JM, Alderson NL, Welsh RS. Serotonin and central nervous system fatigue: nutritional considerations. Am J Clin Nutr 2000; 72: S573-578.

27. Du L, Bakish D, Hrdina PD. Tryptophan hydroxylase gene 218A/C polymorphism is associated with somatic anxiety in major depressive disorder. J Affect Disord 2001; 65: 37-44.

28. Fernstrom JD. Branched-chain amino acids and brain function. J Nutr 2005; 135: S1539-1546.

29. Greer BK, White JP, Arguello EM, Haymes EM. Branched-chain amino acid supplementation lowers perceived exertion but does not affect performance in untrained males. I Strength Cond Res 2011; 25: 539-544.

30. Grrishma B, Gaur G S, Velkumary S, Gurunandan U, Dutt A, Dinesh T. Comparison of hand and foot reaction times among females-a methodological study using recognition auditory 
reaction time. International Journal of Current Research 2013; 5: $4272-4274$

31. Guezennec CY, Abdelmalki A, Serrurier B, Merino D, Bigard X, Berthelot M, Pierard C, Peres M. Effects of prolonged exercise on brain ammonia and amino acids. Int J Sports Med 1998; 19: 323-327.

32. Hamon M, Bourgoin S, Artaud F, El Mestikawy S. The respective roles of tryptophan uptake and tryptophan hydroxylase in the regulation of serotonin synthesis in the central nervous system. J Physiol 1981; 77: 269-279.

33. Haussinger D. Nitrogen metabolism in liver: structural and functional organization and physiological relevance. Biochem J 1990; 267: 281-290.

34. Hellsten Y, Richter EA, Kiens B, Bangsbo J. AMP deamination and purine exchange in human skeletal muscle during and after intense exercise. J Physiol 1999; 3: 909-920.

35. Jalan R, Wright G, Davies NA, Hodges SJ. L-Ornithine phenylacetate (OP): a novel treatment for hyperammonemia and hepatic encephalopathy. Med Hypotheses 2007; 69: 1064-1069.

36. Kilpatrick IC. Rapid, automated HPLC analysis of neuroactive and other amino acids in microdissected brain regions and brain slice superfusates using fluorimetric detection. In: Neuroendocrine research methods. Vol. 2. Greenstein B (ed.). Harwood Academic Publishers, Chur, 1991; pp. 555-578.

37. MacGillivray L, Reynolds KB, Rosebush PI, Mazurek MF. The comparative effects of environmental enrichment with exercise and serotonin transporter blockade on serotonergic neurons in the dorsal raphe nucleus. Synapse 2012; 66: 465-470.

38. Meeusen R, Watson P. Amino acids and the brain: do they play a role in "central fatigue"? Int J Sport Nutr Exerc Metab 2007 17: S37-46.

39. Mikulski T, Ziemba A, Chmura J, Wiśnik P, Kurek Z, Kaciuba Uściłko H, Nazar K. The effect of supplementation with branched chain amino acids (BCAA) on psychomotor performance during graded exercise in human subjects. Biol Sport 2002; 19: 295-301.

40. Nybo L, Dalsgaard MK, Steensberg A, Møller K, Secher NH. Cerebral ammonia uptake and accumulation during prolonged exercise in humans. J Physiol 2005; 563: 285-290.

41. Parise G, Bosman MJ, Boecker DR, Barry MJ, Tarnopolsky MA. Selective serotonin reuptake inhibitors: Their effect on high-intensity exercise performance. Archives of Physical Medicine and Rehabilitation 2001; 82: 867-871.

42. Pérez Hernández JL, Higuera de la Tijera F, Serralde-Zúñiga AE, Abdo Francis JM. Critical analysis of studies evaluating the efficacy of infusion of L-ornithine L-aspartate in clinical hepatic encephalopathy in patients with liver failure. Ann Hepatol. 2011; 10 Suppl 2: S66-69.

43. Pitkanen H, Nykanen T, Knuutinen J. Free amino acid pool and muscle protein balance after resistance exercise. Med Sci Sports Exerc 2003; 35: 784-792.

44. Rees CJ, Oppong K, Al Mardini H, Hudson M, Record CO. Effect of L-ornithine-L-aspartate on patients with and without TIPS undergoing glutamine challenge: a double blind, placebo controlled trial. Gut 2000; 47: 571-574

45. Schmid M, Peck-Radosavljevic M, König F, Mittermaier C, Gangl A Ferenci P. A double-blind, randomized, placebo-controlled trial of intravenous L-ornithine-L-aspartate on postural control in patients with cirrhosis. Liver Int 2010; 30: 574-582.

46. Shephard RJ, Johnson N. Effects of physical activity upon the liver. Eur J Appl Physiol 2015; 115: 1-46.

47. Sikorska H, Cianciara J, Wiercińska-Drapało A. Physiological functions of L-ornithine and L-aspartate in the body and the efficacy of administration of L-ornithine-L-aspartate in conditions of relative deficiency. Pol Merkur Lekarski 2010; 28: 490-495

48. Staedt U, Leweling H, Gladisch R, Kortsik C, Hagmüller E, Holm E. Effects of ornithine aspartate on plasma ammonia and plasma amino acids in patients with cirrhosis. A double-blind, randomized study using a four-fold crossover design. J Hepatol 1993; 19: 424-430.

49. Strüder HK, Hollmann W, Platen P, Donike M, Gotzmann A, Weber K. Influence of paroxetine, branched-chain amino acids and tyrosine on neuroendocrine system responses and fatigue in humans. Horm Metab Res 1998; 30: 188-194.

50. Strüder HK, Weicker H. Physiology and pathophysiology of the serotonergic system and its implications on mental and physical performance. Int J Sports Med 2001; 22: 482-497.

51. Wagenmakers AJ, Coakley JH, Edwards RH. Metabolism of branched-chain amino acids and ammonia during exercise: clues from McArdle's disease. Int J Sports Med 1990; 11 Suppl 2: S101-113.

52. Wagenmakers AJ, Beckers EJ, Brouns F, Kuipers H, Soeters PB, van der Vusse GJ, Saris WH. Carbohydrate supplementation, glycogen depletion, and amino acid metabolism during exercise. Am J Physiol 1991; 260 (6 Pt 1): E883-890.

53. van Wijck K, Lenaerts K, van Loon LJ, Peters WH, Buurman WA, Dejong $\mathrm{CH}$. Exercise-induced splanchnic hypoperfusion results in gut dysfunction in healthy men. PLoS One 2011; 6: e22366.

54. Wiśnik P, Chmura J, Ziemba AW, Mikulski T, Nazar K. The effect of branched chain amino acids on psychomotor performance during treadmill exercise of changing intensity simulating a soccer game. Appl Physiol Nutr Metab 2011; 36: 856-862. 